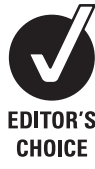

Department of Epidemiology and Public Health, University College London, UK

Correspondence to: Dr M Hamer, Department of Epidemiology and Public Health, University College London, 1-19 Torrington Place, London WC1E 6BT, UK; m.hamer@ucl.ac.uk

Accepted 15 February 2008 Published Online First 10 April 2008

\title{
Dose-response relationship between physical activity and mental health: the Scottish Health Survey
}

\author{
M Hamer, E Stamatakis, A Steptoe
}

\section{ABSTRACT}

Objectives: Regular physical activity is thought to be associated with better mental health, although there is a lack of consensus regarding the optimal amount and type of activity to achieve these benefits. The association between mental health and physical activity behaviours was examined among a representative sample of men and women from the Scottish Health Surveys.

Methods: Self-reported physical activity was measured and the General Health Questionnaire (GHO-12) was administered in order to obtain information on current mental health. Participants were 19842 men and women. Risk estimates per category of physical activity sessions per week were calculated using logistic regression models.

Results: Psychological distress (based on a score of 4 or more on the GHQ-12) was evident in 3200 participants. Any form of daily physical activity was associated with a lower risk of psychological distress after adjustment for age, gender, social economic group, marital status, body mass index, long-standing illness, smoking and survey year (OR 0.59, 95\% Cl 0.52 to 0.66, p<0.001). A doseresponse relationship was apparent, with moderate reductions in psychological distress with less frequent activity (OR $0.67,95 \% \mathrm{Cl} 0.61$ to 0.75 ). Different types of activities including domestic (housework and gardening), walking and sports were all independently associated with lower odds of psychological distress, although the strongest effects were observed for sports (OR 0.67, 95\% Cl 0.54 to 0.82).

Conclusion: Mental health benefits were observed at a minimal level of at least $20 \mathrm{~min} /$ week of any physical activity, although a dose-response pattern was demonstrated with greater risk reduction for activity at a higher volume and/or intensity.

Mental illness is a risk factor for mortality and morbidity and is becoming an increasing health concern. ${ }^{1}$ Regular physical activity is thought to be associated with better mental health. Prospective cohort studies have generally showed inverse associations between physical activity and incident depression $^{2-6}$ and an association between reduced activity and emerging depression, ${ }^{7}$ although others have produced conflicting findings. ${ }^{8-11}$ There is also some evidence from prospective cohorts demonstrating an association between physical activity and lower risk of dementia and cognitive decline. ${ }^{12}$ The results from clinical trials that have examined the effect of exercise on mental health have been generally difficult to interpret because of a lack of good quality studies with adequate follow-up, ${ }^{13}$ although recent studies have begun to address these limitations by employing credible control groups and considering issues such as the confounding effects of psychotherapy. ${ }^{14} 15$

The reason for some of the conflicting findings is largely related to incomplete measurements that did not enquire about all physical activity types, variation in the methods used to assess mental health, insufficient sample sizes and lack of adjustment for possible confounding factors. As a result, the amount and type of activity that is required to achieve mental health benefits has not been clearly established and at present is insufficient to inform public health policy. There is a limited amount of experimental work that has specifically examined the dose-response relationship. Previous work in children and adolescents demonstrated no significant differences when comparing the effects of vigorous with lower intensity exercise interventions on mental health outcomes. ${ }^{16}$ In contrast, Dunn et a ${ }^{17}$ showed that adults assigned to a higher energy expenditure exercise treatment $(17.5 \mathrm{kcal} / \mathrm{kg} /$ week $)$ had greater reductions in depression than a lower dose of $7.0 \mathrm{kcal} / \mathrm{kg} /$ week, although there were no effects of exercise frequency at 12 weeks.

The aim of the present study was therefore to examine the association of different types and amounts of physical activity with current mental health using the 12-item General Health Questionnaire (GHO-12). We used a large sample of men and women from the Scottish Health Surveys (SHS; 1995, 1998, 2003) in which detailed data of self-reported physical activity was available, together with extensive information on other important confounding factors such as disease history and health behaviours.

\section{METHODS}

\section{Study design and participants}

The SHS is a periodic survey (typically every 35 years) that draws a nationally representative sample of the general population living in households. The sample was drawn using multistage stratified probability sampling with postcode sectors selected at the first stage and household addresses selected at the second stage. Stratification was based on geographical areas and not on individual characteristics of the population. Different samples were drawn for each survey. The present analyses included data from 19842 respondents $(53.9 \%$ female) aged 16 and over (mean (SD) age 45.2 (15.5) years) measured in the 1995,1998 or 2003 SHS.

\section{Procedures}

Data were collected in two household visits. During the first visit trained interviewers collected 
Table 1 Characteristics of the cohort with reference to physical activity levels

\begin{tabular}{lllll}
\hline \multirow{2}{*}{ Variable } & \multicolumn{4}{l}{ Physical activity quartiles (no of sessions/week $>\mathbf{2 0}$ min) } \\
\cline { 2 - 5 } & $\leqslant \mathbf{3 . 5}$ & $\mathbf{3 . 5 1 - 5 . 0 0}$ & $\mathbf{5 . 0 1 - 6 . 7 5}$ & $>\mathbf{6 . 7 5}$ \\
\hline Age (years) & $50.7(14.9)$ & $48.6(14.7)$ & $41.9(14.2)$ & $38.9(13.5)^{*}$ \\
Gender (\% men) & 47.4 & 43.3 & 42.9 & 49.5 \\
Marital status (\%) & & & & \\
$\quad$ Married & 59.5 & 57.8 & 57.6 & $51.4^{*}$ \\
$\quad$ Single/never married & 14.9 & 17.2 & 22.6 & $28.2^{*}$ \\
Social economic group (\%) & & & & \\
$\quad$ Professional & 24.2 & 27.0 & 36.4 & $36.5^{*}$ \\
$\quad$ Manual & 33.4 & 28.6 & 26.7 & $24.6^{*}$ \\
Body mass index (kg/m $\left.{ }^{2}\right)$ & $27.0(5.1)$ & $26.6(4.8)$ & $26.2(4.7)$ & $25.8(3.9)^{*}$ \\
GHQ-12 score & $1.9(3.1)$ & $1.5(2.7)$ & $1.3(2.5)$ & $1.2(2.4)^{*}$ \\
Current smokers (\%) & 38.8 & 35.6 & 26.5 & $25.2^{*}$ \\
Long-standing illness (\%) & 52.4 & 42.4 & 34.4 & $30.5^{*}$ \\
$\quad$ Diabetes & 6.5 & 4.6 & 2.1 & $2.1^{*}$ \\
Hypertension & 33.4 & 29.5 & 17.4 & $17.6^{*}$ \\
CVD & 12.1 & 7.6 & 3.7 & $2.3^{*}$ \\
\hline
\end{tabular}

CVD, cardiovascular disease.

Data shown as mean (SD) values or percentages.

${ }^{*} p<0.001$, difference between groups.

self-reported data and measured height and weight. In the second visit nurses enquired about medical history. Detailed information on the survey method can be found elsewhere. ${ }^{18}$

\section{Measurements}

Interviewers took the height and weight measurements and asked demographic (marital status, social class) and healthrelated questions (smoking, physical activity and mental health). Social-occupational class was defined using the Registrar General classification (I/II professional/intermediate, III skilled non-manual or manual, IV/V part skilled/unskilled). Current mental health was assessed from the GHO-12, which is a measure of psychological distress devised for population studies. The questionnaire enquires about general level of happiness, experience of depressive and anxiety symptoms, and sleep disturbance over the last 4 weeks. Interpretation of the answers is based on a 4-point response scale scored using a bimodal method (symptom present: not at all $=0$, same as usual $=0$, more than usual $=1$ and much more than usual $=$ 1). The GHO-12 is a highly validated instrument and has been strongly associated with various psychological disorders such as depression and anxiety. ${ }^{19}$ A score of $\geqslant 4$ was used to define psychological distress according to studies validating the GHO12 against standardised psychiatric interviews..$^{20}$ Physical activity interviews enquired about participation in the 4 weeks prior to the interview (1998 and 2003) or during a typical week (1995). Frequency of participation (for at least $20 \mathrm{~min}$ per occasion) was assessed across three domains of activity: leisure time sports (eg, cycling, swimming, running, aerobics, dancing and ball sports such as football and tennis), walking for any purpose and domestic physical activity (eg, heavy housework, home improvement activities, manual and gardening work). Participants were also asked whether participation in sports made them feel out of breath or sweaty to assess intensity. The validity of the physical activity questions is supported by data on 174 British adults where the output of individually calibrated heart rate monitors (four times over a year for four consecutive days on each occasion) was compared against an early version of the above questions. The SHS questionnaire appeared to be a valid measure of energy expenditure for the total physical activity score for men $(p=0.05)$ and women $(p<0.05)$. A significant correlation was also observed between self-reported activity from the questionnaire and aerobic fitness in men $(p=0.001)$ and women $(p<0.05)$.

\section{Analysis of data}

Physical activity frequency quartiles were derived for the number of sessions per week of total physical activity of any intensity lasting at least $20 \mathrm{~min}$. Three frequency categories $(<1 /$ week, $1-3 /$ week, $\geqslant 4 /$ week $)$ were derived for the three separate activity domains (domestic physical activity, walking and sports). We calculated odds ratios (OR) and $95 \%$ confidence intervals (CI) for the risk of psychological distress per category of physical activity using multiple logistic regression. In multivariate models we mutually adjusted for activity types, age, gender, social-occupational class, marital status (single/ never married, married, widowed, separated/divorced), body mass index category (underweight, normal weight, overweight, obese, morbidly obese), presence of long-standing illness (yes, no), smoking (never, previous, current) and survey year. $\chi^{2}$ and one-way ANOVA tests were used to examine differences in demographic and health-related variables with respect to physical activity quartiles. All analyses were conducted using SPSS Version 14.

\section{RESULTS}

We identified 3200 participants with psychological distress from the GHO-12. Table 1 shows the characteristics of the sample split into total physical activity quartiles. Approximately $32 \%$ of the sample performed none or one session of physical activity per week lasting at least $20 \mathrm{~min}$ after the exclusion of domestic activities. Participants in the higher activity quartiles were more likely to be younger, not married, come from a higher socioeconomic stratum, not smoke, have lower body mass index, lower GHO-12 scores, and less likely to have a longstanding illness.

Table 2 presents logistic regression models for physical activity frequency and risk of psychological distress, based on a GHQ-12 score of $\geqslant 4$. It can be seen that all types of activity were independently associated with lower odds of psychological distress, although a dose-response relationship was only demonstrated with sports and overall activity. Domestic 
Table 2 Logistic regression models for physical activity and risk of psychological distress (GHO-12 score $\geqslant 4$ )

\begin{tabular}{|c|c|c|c|c|}
\hline & $\begin{array}{l}\text { Cases/total N } \\
3200 / 19842\end{array}$ & $\begin{array}{l}\text { Age adjusted } \\
\text { OR }(95 \% \mathrm{Cl})\end{array}$ & $\begin{array}{l}\text { Model } 1 \\
\text { OR (95\% CI) }\end{array}$ & $\begin{array}{l}\text { Model } 2 \\
\text { OR (95\% CI) }\end{array}$ \\
\hline \multicolumn{5}{|c|}{ Domestic activity sessions } \\
\hline$<1 /$ week & $2293 / 13232$ & 1.00 & 1.00 & 1.00 \\
\hline$\geqslant 4 /$ week & $247 / 1708$ & $0.80(0.70$ to 0.92$)$ & $0.80(0.69$ to 0.92$)$ & 0.84 (0.72 to 0.98$)$ \\
\hline Test for trend & & $\mathrm{p}<0.001$ & $\mathrm{p}<0.001$ & $\mathrm{p}<0.001$ \\
\hline \multicolumn{5}{|c|}{ Walking activity sessions } \\
\hline$\geqslant 4 /$ week & $814 / 5518$ & $0.81(0.74$ to 0.89$)$ & $0.79(0.73$ to 0.87$)$ & 0.87 (0.79 to 0.95$)$ \\
\hline Test for trend & & $\mathrm{p}<0.001$ & $p<0.001$ & $p=0.003$ \\
\hline \multicolumn{5}{|c|}{ Sports activity sessions } \\
\hline$<1 /$ week & 2639/15207 & 1.00 & 1.00 & 1.00 \\
\hline 1-3/week & $445 / 3540$ & $0.66(0.59$ to 0.73$)$ & $0.67(0.60$ to 0.74$)$ & $0.76(0.67$ to 0.84$)$ \\
\hline$\geqslant 4 /$ week & $116 / 1095$ & $0.53(0.43$ to 0.64$)$ & $0.56(0.46$ to 0.68$)$ & 0.67 (0.54 to 0.82 ) \\
\hline 5.01-6.75/week & $470 / 3219$ & $0.57(0.51$ to 0.65$)$ & $0.57(0.50$ to 0.66$)$ & 0.67 (0.59 to 0.76$)$ \\
\hline$>6.75 /$ week & $653 / 5051$ & $0.48(0.43$ to 0.54$)$ & $0.49(0.43$ to 0.54$)$ & 0.59 (0.52 to 0.66$)$ \\
\hline Test for trend & & $\mathrm{p}<0.001$ & $\mathrm{p}<0.001$ & $\mathrm{p}<0.001$ \\
\hline
\end{tabular}

Model 1: adjustment for age, gender, social economic group and marital status.

Model 2: adjustment for age, gender, social economic group, marital status, body mass index category, long-standing illness, smoking, survey year and mutual adjustment for all types of activity.

activity and walking had similar levels of risk reduction, in the region of $13-20 \%$, although there was a greater risk reduction for sports activity of approximately $33 \%$ in the highest frequency category. Any type of daily physical activity was associated with the lowest odds of psychological distress (OR $0.59,95 \%$ CI 0.52 to 0.66 ), and a dose-response relationship was evident such that less frequent activity (3.5-5/week) was associated with slightly less risk reduction (OR 0.67, 95\% CI 0.61 to 0.75$)$.

In subanalyses we examined associations among 1293 participants with existing cardiovascular disease (CVD), defined from a clinically confirmed CVD event or angina. In these analyses dose-response associations were again observed. Any type of daily activity was associated with the lowest odds of psychological distress (OR $0.27,95 \%$ CI 0.16 to 0.47 ) and less frequent activity (3.5-5/week) was associated with slightly less risk reduction (OR $0.40,95 \%$ CI 0.30 to 0.55 ).

\section{DISCUSSION}

The main findings from this study demonstrate strong associations between physical activity and reduced odds of psychological distress. The mental health benefits were observed at a minimum physical activity level of at least $20 \mathrm{~min} /$ week of any type of activity. A dose-response pattern was also observed with greater risk reduction at higher activity levels, especially for sports. Our findings relating to the doseresponse relationship between physical activity and mental health are largely consistent with reports from previous population studies, ${ }^{26}{ }^{21}$ although it is difficult to make direct comparisons with our data because of the differences in measures of mental health and assessment of physical activity. For example, in the Harvard Alumni Study, men who expended $1000-2499$ or $\geqslant 2500 \mathrm{kcal} /$ week were $17 \%$ and $28 \%$ less likely to develop clinically diagnosed depression compared with men who expended $<1000 \mathrm{kcal} /$ week. $^{6}$ Australian women who performed 2-3 sessions per week or daily moderate intensity activity had approximately $20 \%$ and $40 \%$ reductions, respectively, in the risk of subclinical depressive symptoms after 5 years of follow-up. ${ }^{2}$

The mental health benefits of physical activity appear to be independent of potential confounding factors such as longstanding illness, obesity and smoking, although inclusion of these covariates reduced the strength of the association. Thus, the protective effects of physical activity may, in part, operate through these risk factors. Indeed, physical activity is associated with a reduced risk of chronic diseases such as CVD, diabetes, hypertension and some cancers. ${ }^{22}$ Exercise is also thought to improve a number of biological risk factors such as dyslipidaemia, glucose intolerance, inflammation and vascular dysfunction, which have been related to mental health disorders such as depression and dementia. ${ }^{23-25}$ Given that heightened responsiveness to daily stressors is a risk factor for psychological morbidity, ${ }^{26}$ physical activity may also improve mental health by reducing biological stress reactivity. ${ }^{27}$

This is the first study to our knowledge that has specifically considered the importance of different activity types in relation to mental health. Stamatakis et $a l^{28}$ recently reported that, in contrast to leisure time activities, domestic activity was not associated with improvements in CVD risk factors which may partly explain why domestic activity contributed less to mental health benefits in the present analyses. Indeed, previous work has also shown a graded dose-response relationship between cardiorespiratory fitness and depressive symptoms, ${ }^{21}$ suggesting that participation in vigorous sports activities that produce greater fitness improvements is most beneficial for mental health. It is, however, possible that the additional benefits gained from participating in sports may have a psychological component, such as fostering social support networks and developing mastery and better coping abilities. In addition, the measurement of domestic activity may be less reliable than for other forms of activity, increasing error variance. 


\section{What is already known on this topic}

- Mental illness such as depression is a risk factor for morbidity and mortality.

- Regular physical activity is thought to be associated with better mental health.

- The amount and type of activity that is required to achieve mental health benefits has not been clearly established.

\section{What this study adds}

- Lower levels of psychological distress were observed at a minimal level of at least $20 \mathrm{~min} /$ week of any physical activity (including domestic, walking or sports activities).

- A dose-response pattern was demonstrated with greater risk reduction for activity at a higher volume and/or intensity.

The limitations of the present study should be recognised. Given the cross-sectional nature of this study, we cannot exclude the possibility that the present results are explained by reverse causality or confounding from unmeasured variables. In particular, co-morbidities that cause functional impairment may have influenced the results because the association between depression and physical disability may be bidirectional. ${ }^{29}{ }^{30}$ However, we attempted to control for long-standing illness in our analyses and we found a strong inverse association between physical activity and psychological distress in participants with existing CVD, suggesting that these associations are not secondary to co-morbidity. Causality, however, remains an issue. Using a co-twin control method, Stubbe et a ${ }^{31}$ recently suggested that the association between exercise participation and higher levels of life satisfaction and happiness was noncausal and mediated by genetic factors that influence both exercise behaviour and well-being. However, further genetic studies are required to confirm these findings. In the present study we did not separately assess the association between physical activity and positive well-being, which appears to be an independent risk factor for health. ${ }^{32}$ A strength of the study is the large sample size of both men and women and the availability of detailed physical activity information covering both recreational and lifestyle (eg, walking, domestic) activity. Further studies should attempt to examine the effects of exercise intensity on mental health using objective measures.

In summary, mental health benefits were observed at a minimal level of at least $20 \mathrm{~min} /$ week of any physical activity, although a dose-response pattern was demonstrated with greater risk reduction for activity at a higher volume and/or intensity.

Funding: The authors receive grant funding from the British Heart Foundation (MH, AS) and the National Institute for Health Research, England (ES). The Scottish Health Survey is funded by the Scottish Executive.

\section{Competing interests: None.}

Patient consent: Participants gave full informed consent to participate in the study and ethical approval was obtained from the London Research Ethics Council.

\section{REFERENCES}

1. Lopez AD, Mathers CD, Ezzati M, et al. Global and regional burden of disease and risk factors, 2001: systematic analysis of population health data. Lancet 2006;367:1747-57.

2. Brown WJ, Ford JH, Burton NW, et al. Prospective study of physical activity and depressive symptoms in middle-aged women. Am J Prev Med 2005;29:265-72.

3. Strawbridge WJ, Deleger $\mathrm{S}$, Roberts RE, et al. Physical activity reduces the risk of subsequent depression for older adults. Am J Epidemiol 2002;156:328-34.

4. Farmer ME, Locke BZ, Moscicki EK, et al. Physical activity and depressive symptoms: the NHANES I epidemiologic follow-up study. Am J Epidemiol 1988;128:1340-51.

5. Camacho TC, Roberts RE, Lazarus NB, et al. Physical activity and depression: evidence from the Alameda County Study. Am J Epidemiol 1991;134:220-31.

6. Paffenbarger RS Jr, Lee IM, Leung R. Physical activity and personal characteristics associated with depression and suicide in American college men. Acta Psychiatr Scand Supp/ 1994;377:16-22.

7. van Gool CH, Kempen Gl, Penninx BW, et al. Relationship between changes in depressive symptoms and unhealthy lifestyles in late middle aged and older persons: results from the Longitudinal Aging Study Amsterdam. Age Ageing 2003;32:81-7.

8. Weyerer $\mathbf{S}$. Physical inactivity and depression in the community. Evidence from the Upper Bavarian Field Study. Int J Sports Med 1992;13:492-6.

9. Kritz-Silverstein D, Barrett-Connor E, Corbeau C. Cross-sectional and prospective study of exercise and depressed mood in the elderly: the Rancho Bernardo study. Am J Epidemiol 2001;153:596-603.

10. Lampinen $\mathbf{P}$, Heikkinen E. Reduced mobility and physical activity as predictors of depressive symptoms among community-dwelling older adults: an eight-year followup study. Aging Clin Exp Res 2003;15:205-11.

11. Cooper-Patrick L, Ford DE, Mead LA, et al. Exercise and depression in midlife: a prospective study. Am J Public Health 1997;87:670-3.

12. Barnes DE, Whitmer RA, Yaffe K. Physical activity and dementia: the need for prevention trials. Exerc Sport Sci Rev 2007;35:24-9.

13. Lawlor DA, Hopker SW. The effectiveness of exercise as an intervention in the management of depression: systematic review and meta-regression analysis of randomised controlled trials. BMJ 2001;322:763-7.

14. Blumenthal JA, Babyak MA, Doraiswamy PM, et al. Exercise and pharmacotherapy in the treatment of major depressive disorder. Psychosom Med 2007;69:587-96.

15. Mutrie N, Campbell AM, Whyte F, et al. Benefits of supervised group exercise programme for women being treated for early stage breast cancer: pragmatic randomised controlled trial. BMJ 2007;334:517.

16. Larun L, Nordheim LV, Ekeland E, et al. Exercise in prevention and treatment of anxiety and depression among children and young people. Cochrane Database Syst Rev 2006;(3):CD004691.

17. Dunn AL, Trivedi MH, Kampert JB, et al. Exercise treatment for depression: efficacy and dose response. Am J Prev Med 2005;28:1-8.

18. Scottish Government Statistics. Scottish Health Survey Publications. http://www scotland.gov.uk/Topics/Statistics/Browse/Health/scottish-health-survey/Publications (accessed November 2007).

19. Goldberg D, Gater R, Sartorius N, et al. The validity of two versions of the GHO in the WHO study of mental illness in general health care. Psychol Med 1997:27:191-7.

20. Goldberg D, Williams P. A user's guide to the General Health Questionnaire. Berkshire: NFER-Nelson Publishing Co, 1988.

21. Galper DI, Trivedi MH, Barlow CE, et al. Inverse association between physical inactivity and mental health in men and women. Med Sci Sports Exerc 2006;38:173-8.

22. O'Donovan G, Biddle S, Blazevich A, et al. Physical activity in the prevention of chronic diseases: the consensus statement of the British Association of Sports and Exercise Sciences. J Sport Sci 2008 (in press).

23. Krishnan KR, Delong M, Kraemer H, et al. Comorbidity of depression with other medical diseases in the elderly. Biol Psychiatry 2002;52:559-88.

24. Joynt KE, Whellan DJ, O'Connor CM. Depression and cardiovascular disease: mechanisms of interaction. Biol Psychiatry 2003;54:248-61.

25. Rosendorff C, Beeri MS, Silverman JM. Cardiovascular risk factors for Alzheimer's disease. Am J Geriatr Cardiol 2007;16:143-9.

26. Kendler KS, Karkowski LM, Prescott CA. Causal relationship between stressful life events and the onset of major depression. Am J Psychiatry 1999;156:837-41.

27. Hamer M, Steptoe A. Association between physical fitness, parasympathetic control, and proinflammatory responses to mental stress. Psychosom Med 2007;69:660-6.

28. Stamatakis E, Hillsdon M, Primatesta P. Domestic physical activity in relationship to multiple CVD risk factors. Am J Prev Med 2007:32:320-7.

29. Ormel J, Vonkorff M, Oldehinkel AJ, et al. Onset of disability in depressed and nondepressed primary care patients. Psychol Med 1999;29:847-53.

30. Ormel J, Rijsdijk FV, Sullivan M, et al. Temporal and reciprocal relationship between IADL/ADL disability and depressive symptoms in late life. J Gerontol B Psychol Sci Soc Sci 2002; 57 :P338-47.

31. Stubbe JH, de Moor MH, Boomsma DI, et al. The association between exercise participation and well-being: a co-twin study. Prev Med 2007:44:148-52.

32. Pressman SD, Cohen S. Does positive affect influence health? Psychol Bull 2005; 131:925-71. 